\title{
ENTROPY METHODS FOR KINETIC MODELS OF TRAFFIC FLOW*
}

\author{
JEAN DOLBEAULT ${ }^{\dagger}$ AND REINHARD ILLNER ${ }^{\ddagger}$
}

\begin{abstract}
In these notes we first introduce logarithmic entropy methods for time-dependent drift-diffusion equations and then consider a kinetic model of Vlasov-Fokker-Planck type for traffic flows. In the spatially homogeneous case the model reduces to a special type of nonlinear driftdiffusion equation which may permit the existence of several stationary states corresponding to the same density. Then we define general convex entropies and prove a convergence result for large times to steady states, even if more than one exists in the considered range of parameters, provided that some entropy estimates are uniformly bounded.
\end{abstract}

Key words. Traffic flow - time-dependent diffusions - drift-diffusion equations - nonlinear friction and diffusion coefficients - entropy method - relative entropy - large time asymptotics

AMS subject classifications. 90B20, 35K55; 35B40, 35B45, 94A17, 70F40, 60J60, 60J70, 92D99

\section{Introduction}

First order models seem well suited to describe certain regimes of traffic flows, in particular single-lane traffic. In comparison, the interest of kinetic approaches is that they allow a better description of the behaviour of the drivers at the individual level $[4,6]$. It has recently been shown in [4] (see also [5]) that coupled equations of Fokker-Planck type with a degenerate nonlinear diffusion term and braking/ acceleration forces incorporating non-trivial lane change probabilities can explain the existence of multiple steady solutions. The model was introduced in [4]; in the spatially homogeneous case, presented in detail in Sections 2 and 3 of this paper, it reduces to a rather unconventional drift-diffusion equation with at least two unusual features: 1) the coefficient functions depend on the average speed $u=u(t)$, which is proportional to the first moment of the solution itself, and 2) the diffusion coefficient vanishes at $v=u$, so the problem is degenerately parabolic. The equation is in particular nonlinear and admits multiple equilibria.

Over the last five years there has been much interest in entropy methods for parabolic equations [1]. For a very complete and thorough treatise of the power of entropy dissipation methods, we refer to [2]. Although some of these techniques were well known in probability theory, it is surprising to see the variety of models which can be tackled with these methods. The main reason for this success in both linear and nonlinear settings is likely resident in the gradient flow properties [7] which are natural for parabolic equations and translate, for example, readily into a general contraction property [3].

The goal of these notes is to apply entropy methods to nonlinear drift-diffusion equations with generally more than one stationary solution; the spatially homogeneous traffic models are the paradigmatic example for this scenario. Because of the mentioned complications inherent in these models (degeneracy, nonlocal dependencies

*Received: September 27, 2002; accepted (in revised version): May 13, 2003. Communicated by Shi Jin.

†Ceremade (UMR CNRS no. 7534), Université Paris, IX-Dauphine, Place de Lattre de Tassigny, 75775 Paris Cédex 16, France (dolbeaul@ceremade.dauphine.fr). Phone: (33) 144054678 ; Fax: (33) 144054599.

${ }^{\ddagger}$ Mathematics and Statistics, University of Victoria, Clearihue Building D240, 3800 Finnerty Road (Ring Road), Victoria, B.C., Canada V8W 3P4 (rillner@math.uvic.ca). Phone: (250) 721-7456; Fax: (250) 721-8962. 
of the coefficients, implicitly time-dependent coefficients) our main result is weaker than those in [2]. Our conclusion is that solutions of the equations must either converge to one of the steady states or that a quantity involved in the definition of an entropy has to diverge. At this point, when convergence occurs, we are unable to obtain convergence rates; in fact, given that the "local equilibria" themselves vary in a priori unpredictable way with $u(t)$, we cannot even make a guess about convergence rates.

We start with considerations on logarithmic entropies for linear diffusions with time-dependent coefficients. The Fokker-Planck traffic model, which is the homogeneous version of the Vlasov-Fokker-Planck model of traffic dynamics introduced in $[4,5]$ is then described and the convergence to a stationary solution is established in the simple, so-called Maxwellian case. A more general family of convex relative entropies, with a time-dependent normalization coefficient, is then introduced in Section 4 and allows us to describe two possible asymptotic regimes for large times: convergence to a stationary solution or large entropy regime. Further considerations on concentration vs convergence are given in the last part of this paper.

2. Linear diffusions and a logarithmic entropy: the fundamental problem

Consider a solution $f=f(t, v)$ of the linear diffusion equation

$$
f_{t}=\left(-B(t, v) f+D(t, v) f^{\prime}\right)^{\prime}, \quad(t, v) \in \mathbb{R}^{+} \times(0,1)
$$

where $f_{t}$ and $f^{\prime}$ respectively denote the derivatives of $f$ with respect to $t$ and $v$. We shall assume that $D$ is nonnegative and such that

$$
C(t, v)=-\int_{0}^{v} \frac{B(t, w)}{D(t, w)} d w
$$

is well defined for any $(t, v) \in \mathbb{R}^{+} \times(0,1)$, and that $(t, v) \mapsto e^{-C(t, v)}$ belongs to $L^{\infty}\left(\mathbb{R}^{+}, L^{1}(0,1)\right)$. Let $f_{0}$ be a nonnegative initial datum for $(2.1)$ and define

$$
\rho=\int_{0}^{1} f_{0}(v) d v .
$$

Denote by $F_{f}$ the flux associated to $f$ :

$$
F_{f}(t, v):=-B(t, v) f(t, v)+D(t, v) f^{\prime}(t, v) .
$$

Under the no-flux boundary conditions

$$
F_{f}(t, 0)=F_{f}(t, 1)=0 \quad \forall t \geq 0,
$$

the total mass is preserved:

$$
\int_{0}^{1} f(t, v) d v=\rho \quad \forall t \geq 0 .
$$

This allows us to define a local equilibrium

$$
g(t, v)=\rho \frac{e^{-C(t, v)}}{\int_{0}^{1} e^{-C(t, w)} d w}
$$


which has zero flux:

$$
F_{g}(t, v)=0 \quad \forall(t, v) \in \mathbb{R}^{+} \times(0,1),
$$

but which is not in general a stationary solution of (2.1), since $g_{t} \equiv 0$ is not granted. We may also replace $C(t, v)$ by $C(t, v)+C_{0}(t)$ for any function $C_{0}(t)$ without changing the values of $g(t, v)$ : the choice of the primitive of $-B / D$ in $(2.2)$ is free. Note that $g$ is the unique minimizer of the functional

$$
f \mapsto \int_{0}^{1} f(\log f+C(t, v)) d v
$$

on $L_{+}^{1}(0,1)$ under the constraint $\int_{0}^{1} f d v=\rho$. However, the entropy production

$$
\frac{d}{d t} \int_{0}^{1} f(\log f+C(t, v)) d v
$$

will contain a term $\int_{0}^{1} f C_{t} d v$, and unless we have good control over the sign of $C_{t}$ we cannot use the standard methods from $[1,2]$ to prove results about asymptotic behaviour. A first attempt to remedy this would be to define the relative entropy $e[t, f]$ by

$e[t, f]:=\int_{0}^{1}(f \log f-g \log g+C(t, v)(f-g)) d v-\iint_{(0,1) \times(0, t)} C_{t}(s, v)(f-g)(s, v) d v d s$.

A straightforward computation then shows that if $f$ is a solution of (2.1) and if all above conditions are fulfilled, then

$$
\frac{d}{d t} e[t, f(t, .)]=-\int_{0}^{1} D(t, v) f\left|\frac{f^{\prime}}{f}-\frac{g^{\prime}}{g}\right|^{2} d v .
$$

This is an entropy production term of the desired kind, but the computation is formal and yields no conclusions, since now we have in general no control on any lower bound of $e[t, f(t,)$.$] , especially when we shall generalize (2.1) to a nonlinear model in which$ the coefficients $B$ and $D$ depend on $f$ through the mean velocity.

\section{The homogeneous Fokker-Planck model of traffic dynamics}

The homogeneous Fokker-Planck model of traffic dynamics as presented in [4] corresponds to Eq. (2.1) in the case $\rho \in(0,1]$ and where $B$ and $D$ are defined in a nonlinear way as functions of the average velocity

$$
u(t)=\frac{1}{\rho} \int_{0}^{1} v f(t, v) d v
$$

as follows:

$$
B(t, v)= \begin{cases}-C_{B}|v-u(t)|^{2} \rho\left(1-\left|\frac{v-u(t)}{1-u(t)}\right|^{\delta}\right) & \text { if } v>u(t) \\ C_{A}|v-u(t)|^{2}(1-\rho) & \text { if } v \leq u(t)\end{cases}
$$

and

$$
D(t, v)=\sigma m_{1}(\rho) m_{2}(u(t))|v-u(t)|^{\gamma}
$$


for some positive constants $C_{A}, C_{B}, \sigma$ and nonnegative exponents $\gamma$ and $\delta$. In [4] $m_{2}$ was chosen as $m_{2}(u)=u(1-u)$, and $m_{1}$ a function which consists of two linked pieces of Gaussian distributions such that $\max _{(0,1)} m_{1}=m_{1}(0.3)$ and $m_{1}(0)=m_{1}(1)=0$. In the rest of this paper (except in Section 6), we will assume that $\gamma<3$, so that $C$ is well defined by $(2.2)$ and uniformly bounded w.r.t. $(t, v) \in \mathbb{R}_{+} \times(0,1)$ as long as $m_{2}(u(t))$ is bounded away from 0 . For these choices the following result has been obtained in [4]. We also refer to [5] for further comments on the model.

Proposition 1. [4] Any stationary solution is uniquely determined by $\rho$ and its average velocity $u$. The set of stationary solutions is therefore represented by a diagram $(\rho, u[\rho])$, which is in general multivalued. For any $\rho \in(0,1]$, there exists at least one stationary solution. For some parameter choices (for example $\gamma=\delta=1$; see [4]), there exist $\rho_{1}, \rho_{2} \in(0,1)$ such that there is exactly one solution for $\rho \in(0,1) \backslash\left(\rho_{1}, \rho_{2}\right)$ and three solutions for $\rho \in\left(\rho_{1}, \rho_{2}\right)$.

Example. The Maxwellian case. This is a special case for which the technique of Section 2 works after a further modification. Assume that $u, B$ and $D$ are defined by (3.1), (3.2) and (3.3) with $\delta=\infty, C_{B} \rho=C_{A}(1-\rho):=K, m_{2} \equiv 1$ and $\gamma=1$. Then $B=K|u-v|(u-v)$,

$$
C=\frac{K}{2 \sigma m_{1}(\rho)}|u-v|^{2}+C_{0}(t)
$$

and

$$
\int_{0}^{1} C_{t}(t, v)(f-g) d v=\left(C_{0}\right)_{t} \int_{0}^{1}(f-g) d v+\frac{K}{\sigma m_{1}(\rho)} \frac{d u}{d t} \cdot \int_{0}^{1}(v-u(t))(f-g) d v .
$$

On the one hand, $\int_{0}^{1}(v-u(t)) f d v=0$ by the definition of $u(t)$, and on the other hand, since $g$ is itself a function of $v-u(t)$, there exists a function

$$
F(u):=\log \left(\int_{0}^{1} \exp \left\{-\frac{K}{\sigma m_{1}(\rho)}|v-u(t)|^{2}\right\} d v\right)
$$

such that

$$
\frac{d}{d t} F(u(t))=F^{\prime}(u(t)) \frac{d u}{d t}=\frac{K}{\sigma m_{1}(\rho)} \frac{d u}{d t} \int_{0}^{1}(v-u(t)) g d v,
$$

Therefore, if we define

$$
e[t, f]:=\int_{0}^{1}(f \log f-g \log g+C(t, v)(f-g)) d v-F(u(t)) .
$$

and observe that $F$ is bounded for $u(t) \in[0,1], e$ has natural lower bounds, and the entropy production term is nonpositive by contruction.

For this Maxwellian example the steady states are unique (this is not hard to see - for a simple argument see [5]). By using a reasoning as in Section 5 below one can prove that $f(t, \cdot)$ converges as $t \rightarrow+\infty$ to this unique stationary solution of (2.1) which is of the form (2.3) for some unique $u=u[\rho]$.

We sketch the main steps of the proof. Since $\log g=-C+$ Constant,

$$
\begin{aligned}
\int_{0}^{1}(f \log f-g \log g+C(t, v)(f-g)) d v & =\int_{0}^{1} f \log \left(\frac{f}{g}\right) d v \\
& \geq \int_{0}^{1} f d v \log \left(\frac{\int_{0}^{1} f d v}{\int_{0}^{1} g d v}\right)=0
\end{aligned}
$$


by Jensen's inequality: $e[t, f]$ is therefore bounded from below and converges to some limit as $t \rightarrow+\infty$, which means that $\int_{0}^{1} D(t, v) f\left|f^{\prime} / f-g^{\prime} / g\right|^{2} d v$ converges to zero. This is enough to prove the strong convergence of $u(t)$ to $u_{\infty}(t)=\frac{1}{\rho} \int_{0}^{1} v f_{\infty}(t, v) d v$, where $f_{\infty}$ is the weak $L^{1}$ limit of $\left.f\left(\cdot+t_{n}, \cdot\right)\right)$ as $t_{n} \rightarrow+\infty$ (the limit is uinque). It is then easy to conclude that $f_{\infty}=g$ is a stationary solution.

This approach generalizes to any equation of type (2.1) such that

$$
\frac{B}{D}=-k(v-u(t)),
$$

for some positive constant $k$, whatever $D$ is. The specific form of $D$ then plays a crucial role if one wants to determine the rate of convergence to the stationary solution.

\section{Convex entropies}

In this section we are going to consider more general entropies than in Section 2, which apply to (2.1) both in the linear and in the nonlinear case corresponding to the traffic model described in Sections 2 and 3 respectively. Define the relative entropy of $f$ with respect to $g$ by

$$
E[f \mid g]=\int_{0}^{1} \Phi\left(\frac{f}{g}\right) g d v
$$

for some convex function $\Phi$. Our "standard" example is $\Phi_{\alpha}(x)=\left(x^{\alpha}-x\right) /(\alpha-1)$ for some $\alpha>1$. We recover logarithmic entropies by taking $\Phi(x)=x \log x$ (this corresponds to a limit case that we shall denote by " $\alpha=1$ "). Both examples satisfy in addition $\Phi(0)=0$. Taking into account the boundary conditions, we can rewrite $(2.1)$ as follows:

$$
\begin{cases}f_{t}=\left[D(t, v) f\left(\frac{f^{\prime}}{f}-\frac{g^{\prime}}{g}\right)\right]^{\prime}=\left[D(t, v) g\left(\frac{f}{g}\right)^{\prime}\right]^{\prime} & \forall(t, v) \in \mathbb{R}^{+} \times(0,1) \\ \left(\frac{f}{g}\right)^{\prime}(t, v)=0 & \forall t \in \mathbb{R}^{+}, v=0,1\end{cases}
$$

where $g=g(t, v)$ is given by

$$
g(t, v)=\kappa(t) e^{-C(t, v)}
$$

for some $\kappa(t) \neq 0$ to be chosen later (and not given by (2.3) any more). With $f$ and $g$ given by (4.2) and (4.3) respectively,

$$
\frac{d}{d t} E[f(t, \cdot) \mid g(t, \cdot)]=\int_{0}^{1} \Phi^{\prime}\left(\frac{f}{g}\right) f_{t} d v+\int_{0}^{1}\left[\Phi\left(\frac{f}{g}\right)-\frac{f}{g} \Phi^{\prime}\left(\frac{f}{g}\right)\right] g_{t} d v .
$$

Using (4.2), we can evaluate the first term of the r.h.s. by

$$
\int_{0}^{1} \Phi^{\prime}\left(\frac{f}{g}\right) f_{t} d v=-\int_{0}^{1} \Phi^{\prime \prime}\left(\frac{f}{g}\right) D(v, t) g\left|\left(\frac{f}{g}\right)^{\prime}\right|^{2} d v .
$$

Let $\Psi(x):=\Phi(x)-x \Phi^{\prime}(x)<0$, for any $x>0$ (this is true for our standard examples and for any strictly convex $\Phi$ such that $\Phi(0)=0)$. The second term is

$$
\begin{aligned}
\int_{0}^{1}\left[\Phi\left(\frac{f}{g}\right)-\frac{f}{g} \Phi^{\prime}\left(\frac{f}{g}\right)\right] g_{t} d v & =\int_{0}^{1} \Psi\left(\frac{f}{g}\right) g \frac{g_{t}}{g} d v \\
& =\int_{0}^{1} \Psi\left(\frac{f}{g}\right) g\left(\frac{\dot{\kappa}}{\kappa}-C_{t}(t, v)\right) d v
\end{aligned}
$$


where we used the notation $\dot{\kappa}=\frac{d \kappa}{d t}$. The right hand side vanishes if we choose

$$
\left\{\begin{array}{l}
\dot{\kappa}=\kappa \frac{\int_{0}^{1} \Psi\left(\frac{f}{g}\right) g C_{t}(t, v) d v}{\int_{0}^{1} \Psi\left(\frac{f}{g}\right) g d v} \\
\kappa(0)=1 .
\end{array}\right.
$$

Note that $C$ depends on $u(t)$ and therefore on $f$, even in the standard cases $\Phi_{\alpha}(x)=$ $\left(x^{\alpha}-x\right) /(\alpha-1)$ or $\Phi(x)=x \log x(\alpha=1)$. In the limit case $\alpha=1, g$ is not directly involved in the definition of $\kappa$ :

$$
\dot{\kappa}=\frac{\kappa}{\rho} \int_{0}^{1} f(t, v) C_{t}(t, v) d v
$$

since $\Psi(x)=-x$ is linear and it is clear that $\kappa$ is globally defined and positive if $C_{t}$ is (locally in $t$ ) bounded. For the other cases, we use the following result.

Lemma 4.1. Consider a solution of (2.1). Assume that $f$ and $C$ are smooth and globally defined in $t$. If $\Phi$ is convex, superlinear at $+\infty$ and such that $\Psi(x)=\Phi(x)-$ $x \Phi^{\prime}(x)$ has no zero for $x>0$, then $\kappa$ is bounded away from zero. If moreover $C_{t}$ is, locally in $t$, bounded, then (4.4) has a global positive solution.

Proof. While $\kappa$ is positive and well defined, we have by Jensen's inequality

$$
\kappa(t) \int_{0}^{1} e^{-C} d v \Phi\left(\frac{\rho}{\kappa(t) \int_{0}^{1} e^{-C} d v}\right) \leq E[f(t, \cdot) \mid g(t, \cdot)] \leq E[f(0, \cdot) \mid g(0, \cdot)] .
$$

Because of the superlinearity of $\Phi$, this is possible if and only if $\kappa(t)$ is bounded away from zero. On the other hand, with $C_{t}$ locally bounded in $t$,

$$
\frac{d}{d t}(\log \kappa) \leq\left\|C_{t}(t, \cdot)\right\|_{L^{\infty}(0,1)},
$$

which proves that $\kappa$ is locally bounded in t.

Forthechoiceof $\kappa$ corresponding to (4.4), $E[f(t, \cdot) \mid g(t, \cdot)]$ is bounded from below by Jensen's inequality (4.5) as long as $\kappa$ is finite, and nonincreasing :

$$
\frac{d}{d t} E[f(t, \cdot) \mid g(t, \cdot)]=-\int_{0}^{1} \Phi^{\prime \prime}\left(\frac{f}{g}\right) D(v, t) g\left|\left(\frac{f}{g}\right)^{\prime}\right|^{2} d v=:-I[f(t, \cdot) \mid g(t, \cdot)] .
$$

For the convenience of the reader we give the explicit form of the entropy and the entropy production terms in the standard cases.

Lemma 4.2. Let $f$ be a smooth function on $(0,1)$.

(i) if $\Phi_{\alpha}(x)=\left(x^{\alpha}-x\right) /(\alpha-1)$ for some $\alpha>1$, then $E[f \mid g]=E_{\alpha}[f \mid g]$ is given by

$$
E_{\alpha}[f \mid g]=\frac{1}{\alpha-1} \int_{0}^{1}\left(f^{\alpha} g^{1-\alpha}-f\right) d v=\frac{\kappa^{1-\alpha}}{\alpha-1} \int_{0}^{1} f^{\alpha} e^{(\alpha-1) C(t, v)} d v-\frac{\rho}{\alpha-1},
$$

(ii) if $\Phi(x)=x \log x$, then

$$
E[f \mid g]=\int_{0}^{1} f \log \left(\frac{f}{g}\right) d v=\int_{0}^{1} f(\log f+C(t, v)-\log \kappa) d v
$$


and in both cases,

$$
\begin{aligned}
I[f \mid g] & =\alpha \int_{0}^{1} D f^{\alpha-2} g^{3-\alpha}\left|\left(\frac{f}{g}\right)^{\prime}\right|^{2} d v \\
& =\alpha \int_{0}^{1} D f^{\alpha} g^{1-\alpha}\left|\frac{f^{\prime}}{f}-\frac{g^{\prime}}{g}\right|^{2} d v \\
& =\frac{4}{\alpha} \int_{0}^{1} D g^{1-\alpha}\left|\left(f^{\alpha / 2}\right)^{\prime}+\frac{\alpha}{2} C^{\prime} f^{\alpha / 2}\right|^{2} d v .
\end{aligned}
$$

We summarize our observations as

Proposition 2. Assume that $\Phi$ is convex, superlinear at $+\infty$ and that $\Psi(x):=$ $\Phi(x)-x \Phi^{\prime}(x)$ has no zero. Assume that $C_{t}$ is bounded in $L^{\infty}((0, T) \times(0,1))$ for any $T>0$, and consider a smooth solution $f$ of (4.2), with $E, g$, and $\kappa$ defined by (4.1), (4.3) and (4.4). Then

$$
E[f(t, \cdot) \mid g(t, \cdot)]+\int_{0}^{t} \int_{0}^{1} \Phi^{\prime \prime}\left(\frac{f}{g}\right) D(v, s) g\left|\left(\frac{f}{g}\right)^{\prime}\right|^{2} d v d s=E[f(0, \cdot) \mid g(0, \cdot)] .
$$

As a consequence, with $E[f(0, \cdot) \mid g(0, \cdot)]=: \mathcal{E}_{0}$,

(i) if $\Phi_{\alpha}(x)=\left(x^{\alpha}-x\right) /(\alpha-1)$ for some $\alpha>1$, then

$$
\frac{4}{\alpha} \int_{0}^{t} \int_{0}^{1} D g^{1-\alpha}\left|\left(f^{\alpha / 2}\right)^{\prime}+\frac{\alpha}{2} C^{\prime} f^{\alpha / 2}\right|^{2} d v d s+\frac{(\kappa(t))^{1-\alpha}}{\alpha-1} \int_{0}^{1} f^{\alpha} e^{(\alpha-1) C} d v=\mathcal{E}_{0}+\frac{\rho}{\alpha-1},
$$

(ii) if $\Phi(x)=x \log x$ (case $\alpha=1)$, then

$$
4 \int_{0}^{t} \int_{0}^{1} D\left|(\sqrt{f})^{\prime}-\frac{1}{2} \sqrt{f} \frac{g^{\prime}}{g}\right|^{2} d v d s+\int_{0}^{1} f(t, v) \log \left(\frac{f(t, v)}{\kappa(t) e^{-C(t, v)}}\right) d v=\mathcal{E}_{0} .
$$

The results of Lemma 4.1 and Proposition 2 apply in particular to the nonlinear model of Section 3, provided that $u(t)$ stays away from the limit values $u=0$ and $u=1$. Note that the mass conservation implies that if $u$ converges to one of these limit values, then $f$ must approach the Dirac $\delta$ function supported at this value, i.e., $f$ forms concentrations. The choice of $m_{2}$ specified earlier allows this possibility. The assumptions on $C$ made in the Proposition include the implicit assumption that $u$ does not approach 0 or 1 .

The main difficulty to obtain information on the large time behaviour is to replace local estimates by global ones (see Remark 5).

\section{Large time behaviour}

Assuming that there exists a global smooth solution of (2.1) in the nonlinear case where $u, B$ and $D$ are given by (3.1), (3.2) and (3.3), we are going to describe two possible asymptotic regimes.

5.1. Convergence to a stationary solution. In this paragraph, we assume that

$$
\limsup _{t \rightarrow+\infty} \kappa(t)<+\infty
$$


For simplicity, we will consider only entropies corresponding to the "standard case" of Section 4. However, there is a further restriction, namely $\alpha \leq 2$, in order to control the mean velocity. The next theorem is our main result.

Theorem 3. Assume that $\Phi_{\alpha}(x)=\left(x^{\alpha}-x\right) /(\alpha-1)$ with $\alpha \in(1,2]$ and $\Phi(x)=x \log x$ in the limit case $\alpha=1$. Consider a smooth global in time solution $f$ of (2.1) with $u, B$ and $D$ depending nonlinearly on $f$ according to (3.1), (3.2) and (3.3), for some smooth function $m_{2}$. Let $\rho=\int_{0}^{1} f(t, v) d v \in(0,1]$. Assume that $E[f \mid g]$ is well defined and $C^{1}$ in $t$, with $E, g$, and $\kappa$ defined by (4.1), (4.3) and (4.4). Assume furthermore that $\gamma<3$ and that $\limsup _{t \rightarrow \infty} \kappa(t)<\infty$. If there exists an $\varepsilon \in\left(0, \frac{1}{2}\right)$ such that

$$
\varepsilon<u(t)=\frac{1}{\rho} \int_{0}^{1} v f(t, v) d v<1-\varepsilon \quad \forall t>0,
$$

then, as $t \rightarrow+\infty, f(t, \cdot)$ converges a.e. to a stationary solution $f_{\infty}$ of (2.1) where $\int_{0}^{1} f_{\infty} d v=\rho, u_{\infty}:=\frac{1}{\rho} \int_{0}^{1} v f_{\infty}(t, v) d v=u[\rho]$, with the notations of Proposition 1.

REMARK 4 . If the assumption on $u$ was not satisfied, there would exist a $t_{0} \in(0,+\infty]$ such that $\lim _{t \rightarrow t_{0}} u(t)=0$ or 1 . This would be possible only if concentration occurs, i.e., if $f(t, \cdot) \rightarrow \rho \delta_{v=0}$ or $\rho \delta_{v=1}$ as $t \rightarrow t_{0}$, where $\delta_{v=0}$ and $\delta_{v=1}$ are Dirac $\delta$ functions centered at $v=0$ and $v=1$ respectively. The purpose of Theorem 3 is to study the case where no concentration occurs and to characterize the large time limit in this situation.

Proof. As $\gamma<3$ and as $u(t)$ stays bounded away from 0 and 1 , there is a constant $R>0$ such that for any $(t, v) \in \mathbb{R}^{+} \times(0,1), C(t, v) \in[-R, R]$. Because of Lemma 4.1, there is a $\kappa_{0}>0$ such that $\inf _{t>0} \kappa(t)>\kappa_{0}$. This proves that $g$ is bounded from above and from below by two positive constants.

REMARK 5. Unfortunately we have no information whether or not $\kappa(t)$ will always stay bounded from above; hence the extra assumption that $\lim _{\sup _{t \rightarrow \infty}} \kappa(t)<\infty$. The asymptotic behaviour of $\kappa$ is related to the behaviour of $C_{t}$, over which we have not been able to obtain sufficient control. If $\lim _{\sup _{t \rightarrow \infty}} \kappa(t)=\infty$ then the decrease of $E[f \mid g]$ is consistent with the formation of concentrations in $f(t, \cdot)$ (see the next subsection). Whether such concentrations actually arise is a question which we cannot answer at the present time. The assumptions made in the theorem exclude them.

Consider an increasing unbounded sequence $\left(t_{n}\right)_{n \in \mathbb{N}}$. At each step, one may extract subsequences, which we shall again denote by $\left(t_{n}\right)_{n \in \mathbb{N}}$. Let

$$
f_{n}(t, v)=f\left(t+t_{n}, v\right) \quad \forall(t, v) \in(0,1)^{2} .
$$

The sequence $\left(f_{n}\right)_{n \in \mathbb{N}}$ is bounded in $L^{\infty}\left((0,1 ; d t), L^{1}(0,1 ; d v)\right)$; by Proposition2, $\left(E\left[f_{n} \mid g_{n}\right]\right)_{n \in \mathbb{N}}$, with $g_{n}=g\left(\cdot+t_{n}, \cdot\right)$, is also bounded in $L^{\infty}(0,1 ; d t)$, so that $\left(f_{n}\right)_{n \in \mathbb{N}}$ (resp. $\left.\left(f_{n} \log f_{n}\right)_{n \in \mathbb{N}}\right)$ is bounded in $L^{\infty}\left((0,1 ; d t), L^{1} \cap L^{\alpha}(0,1 ; d v)\right)$ if $\alpha \in(1,2]$ (resp. in $L^{\infty}\left((0,1 ; d t), L^{1}(0,1 ; d v)\right)$ if $\left.\alpha=1\right)$. Thus $\left(f_{n}\right)_{n \in \mathbb{N}}$ is weakly relatively compact in $L^{1} \cap L^{\alpha}\left((0,1)^{2}\right)$ for any $\alpha \in[1,2]$. Denote by $f_{\infty}$, the weak limit of $\left(f_{n}\right)_{n \in \mathbb{N}}$, up to the extraction of a subsequence. Notice that $f_{\infty}$ is possibly $t$ dependent. Let $u_{n}:=\frac{1}{\rho} \int_{0}^{1} v f_{n} d v$. At least * weakly in $L^{\infty}(0,1 ; d t), u_{n}$ converges to $u_{\infty}:=\frac{1}{\rho} \int_{0}^{1} v f_{\infty} d v$. According to Lemma 4.1 and Proposition 2,

$$
\lim _{n \rightarrow \infty} \int_{0}^{1} \int_{0}^{1} D_{n}(t, v)\left|\left(f_{n}^{\alpha / 2}\right)^{\prime}-\frac{\alpha}{2} f_{n}^{\alpha / 2} \frac{g_{n}^{\prime}}{g_{n}}\right|^{2} d v d t=0
$$


where $D_{n}(t, v)=D\left(t+t_{n}, v\right)=\sigma m_{1}(\rho) m_{2}\left(u_{n}(t)\right)\left|v-u_{n}(t)\right|^{\gamma}$ depends on $f_{n}$ through $u_{n}$. Note that by developing the square, since

$$
\int_{0}^{1} \int_{0}^{1} D_{n}(t, v)\left|f_{n}^{\alpha / 2} \frac{g_{n}^{\prime}}{g_{n}}\right|^{2} d v d t
$$

is bounded by the entropy itself,

$$
\int_{0}^{1} \int_{0}^{1} D_{n}(t, v)\left|\left(f_{n}^{\alpha / 2}\right)^{\prime}\right|^{2} d v d t
$$

is also uniformly bounded. If we multiply (2.1) by $v$ and integrate with respect to $v$, we get

$$
\rho \frac{d u_{n}}{d t}=\int_{0}^{1} v \partial_{t} f_{n}(t, v) d v=\int_{0}^{1} B\left(t+t_{n}, v\right) f_{n}(t, v) d v-\int_{0}^{1} D_{n}(t, v) f_{n}^{\prime}(t, v) d v .
$$

The first term of the right hand side is clearly bounded in $L^{\infty}(0,1 ; d t)$. As for the second one, we can bound it in $L^{2}(0,1 ; d t)$ using the Cauchy-Schwarz inequality:

$$
\begin{aligned}
\int_{0}^{1} D_{n} f_{n}^{\prime} d v= & \frac{2}{\alpha} \int_{0}^{1} \sqrt{D_{n}}\left(f_{n}^{\alpha / 2}\right)^{\prime} \cdot \sqrt{D_{n}} f_{n}^{1-\alpha / 2} d v \\
& \leq \frac{2}{\alpha}\left[\int_{0}^{1} D_{n}\left|\left(f_{n}^{\alpha / 2}\right)^{\prime}\right|^{2} d v \cdot \int_{0}^{1} D_{n} f_{n}^{2-\alpha} d v\right]^{1 / 2} .
\end{aligned}
$$

For $\alpha=2, \int_{0}^{1} D_{n} f_{n}^{2-\alpha} d v$ is bounded by a constant, and for any $\alpha \in[1,2)$, by Hölder's inequality

$$
\int_{0}^{1} D_{n} f_{n}^{2-\alpha} d v \leq\left(\int_{0}^{1} D_{n} f_{n} d v\right)^{2-\alpha}\left(\int_{0}^{1} D_{n} d v\right)^{\alpha-1}
$$

is also bounded by

$$
\rho^{2-\alpha}\left\|D_{n}\right\|_{L^{\infty}\left((0,1)^{2}\right)} \leq \sigma \rho^{2-\alpha} m_{1}(\rho)\left\|m_{2}\right\|_{L^{\infty}(0,1)} .
$$

By these estimates, $d u_{n} / d t$ is bounded uniformly in $n$ in $L^{2}(0,1 ; d t)$. Applying ArzelaAscoli's lemma, we obtain the uniform convergence of $u_{n}$ to $u_{\infty}$, up to the extraction of a subsequence. With $D_{\infty}(t, v)=\sigma m_{1}(\rho) m_{2}\left(u_{\infty}(t)\right)\left|v-u_{\infty}(t)\right|^{\gamma}$, this means that $\left(f_{n}^{\alpha / 2}\right)_{n \in \mathbb{N}}$ actually weakly converges in $L^{\infty}\left((0,1 ; d t), H^{1}\left(0,1 ; D_{\infty}(t, v) d v\right)\right)$.

Up to the extraction of a subsequence, we may assume that $\left(f_{n}^{\alpha / 2}\right)_{n \in \mathbb{N}}$ strongly converges in $L^{2}\left((0,1 ; d t)\left(0,1 ; D_{\infty}(t, v) d v\right)\right)$, and a.e., to $f_{\infty}$.

Using again Proposition 2 , we get

$$
\lim _{n \rightarrow \infty} \int_{0}^{1} \int_{0}^{1} D_{n}(t, v) g_{n}^{1-\alpha}\left|\left(f_{n}^{\alpha / 2}\right)^{\prime}+\frac{\alpha}{2} C^{\prime}\left(t+t_{n}, v\right) f_{n}^{\alpha / 2}\right|^{2} d v d t=0,
$$

where $C^{\prime}\left(t+t_{n}, v\right)$ now strongly converges to a function $C_{\infty}^{\prime}(t, v)$ which depends on $t$ through $u_{\infty}$. Passing to the limit, we obtain

$$
\left(f_{\infty}^{\alpha / 2}\right)^{\prime}+\frac{\alpha}{2} C_{\infty}^{\prime}(t, v) f_{\infty}^{\alpha / 2}=0
$$


so that at least in the sense of distributions, one has:

$$
f_{\infty}(t, v)=\rho \frac{e^{-C_{\infty}(t, v)}}{\int_{0}^{1} e^{-C_{\infty}(t, w)} d w} \quad \forall(t, v) \in(0,1)^{2} \text { a.e. }
$$

To complete the proof, it remains to check that such a steady solution does not depend on $t$ and is unique, (i.e., independent of the initial sequence $\left(t_{n}\right)_{n \in \mathbb{N}}$ ). Because of the uniform convergence of $u_{n}$ to $u_{\infty}, f_{\infty}$ is a solution of (2.1). With the notations of Section 2 and because of (5.1),

$$
\partial_{t} f_{\infty}=\left(F_{f_{\infty}}\right)^{\prime}=0 .
$$

The uniqueness is once more a consequence of Proposition 2. Assume that we can find two sequences $\left(t_{n}^{1}\right)_{n \in \mathbb{N}}$ and $\left(t_{n}^{2}\right)_{n \in \mathbb{N}}$ with $t_{n}^{1}<t_{n}^{2}$ for which the limiting distribution functions are two functions $f_{\infty}^{1}$ and $f_{\infty}^{2}$. Up to extraction of subsequences, we would find

$$
\lim _{n \rightarrow \infty} \int_{t_{n}^{1}}^{t_{n}^{2}+1} \int_{0}^{1} D(v, t) g^{1-\alpha}\left|\left(f^{\alpha / 2}\right)^{\prime}+\frac{\alpha}{2} C^{\prime}(t, v) f^{\alpha / 2}\right|^{2} d v d t=0,
$$

with $\lim _{n \rightarrow \infty}\left(t_{n}^{2}-t_{n}^{1}\right)=+\infty$. Then $\left(f\left(\cdot+t_{n}^{1}, \cdot\right)\right)_{n \in \mathbb{N}}$ would converge for the same reasons as above to both $f_{\infty}^{1}$ and $f_{\infty}^{2}$, which are therefore equal.

5.2. Observations concerning the large entropy regime. As before, we let $\Phi_{\alpha}(x)=\left(x^{\alpha}-x\right) /(\alpha-1)$ for $\alpha>1$ and $\Phi(x)=x \log x$ for $\alpha=1$. We first note an important difference between the entropies $E_{\alpha}[f \mid g]$ and $E[f \mid g](\alpha=1)$. It is transparent from the representation (4.6) that $E_{\alpha}[f \mid g] \geq-\frac{\rho}{\alpha-1}$, which is a fixed lower bound. On the other hand, the term $-\rho \log \kappa(t)$ in (4.7) shows that $E[f \mid g]$ has no automatic lower bound. In what follows, $f$ is assumed to be a global smooth solution of (4.2) and $g$ is a local equilibrium given by (4.3).

THEOREM 6. Let $\alpha>1$ and suppose that there exists a $d>0$ such that

$$
E_{\alpha}[f \mid g]+\frac{\rho}{\alpha-1} \geq d .
$$

Then, if $\limsup _{t \rightarrow+\infty} \kappa(t)=+\infty$, there is a sequence $\left(t_{n}\right)_{n \in \mathbb{N}}$ with $\lim _{n \rightarrow+\infty} t_{n}=$ $+\infty$ and $\lim _{n \rightarrow+\infty} \kappa\left(t_{n}\right)=+\infty$ such that as $n \rightarrow+\infty$,

$$
\int_{0}^{1}\left(f\left(t_{n}, v\right)\right)^{\alpha} e^{(\alpha-1) C\left(t_{n}, v\right)} d v \sim d\left(\kappa\left(t_{n}\right)\right)^{\alpha-1} .
$$

Proof. In view of (4.6), this is immediate because by Proposition 2

$$
E_{\alpha}\left[f_{0} \mid g_{0}\right] \geq E_{\alpha}[f \mid g] \geq d-\frac{\rho}{\alpha-1} .
$$

If there is no $d>0$ such that (5.2) holds, we cannot draw any conclusion whether or not concentration in $f$ will occur. By Jensen's inequality we can prove a lower bound

$$
\int_{0}^{1} f^{\alpha} e^{(\alpha-1) C} d v \geq \rho^{\alpha}\left(\int_{0}^{1} e^{-C} d v\right)^{1-\alpha}
$$


but this inequality yields no information about concentrations. In the case $\alpha=1$ there is no lower bound for $E[f \mid g]$ if $\kappa(t) \rightarrow \infty$. Jensen's inequality yields

$$
E[f \mid g] \geq\left(\int_{0}^{1} f d v\right) \log \left(\frac{\int_{0}^{1} f d v}{\int_{0}^{1} g d v}\right)=\rho \log \rho-\rho \log \left(\int_{0}^{1} e^{-C} d v\right)-\rho \log \kappa(t)
$$

but again this yields no information about concentrations.

REMARK 7. If concentrations occur in $L^{\alpha}(\alpha>1)$ then they have to occur in any $L^{p}$ norm, $p>\alpha$. In the case $\alpha=1$, if concentrations do occur such that $\int_{0}^{1} f \log f d v \rightarrow$ $\infty$, then also $\int_{0}^{1} f^{p} d v \rightarrow \infty$. In fact for any measurable set $A$, by differentiating the logarithm of Hölder's inequality

$$
\log \left(\int_{A} f^{q} d v\right) \leq \frac{p-q}{p-1} \log \left(\int_{A} f d v\right)+\frac{q-1}{p-1} \log \left(\int_{A} f^{p} d v\right), \quad 1<q<p,
$$

at $q=1$, we find

$$
\log \left(\int_{A} f^{q} d v\right) \leq \frac{p-q}{p-1} \log \left(\int_{A} f d v\right)+\frac{q-1}{p-1} \log \left(\int_{A} f^{p} d v\right), \quad 1<q<p
$$

(this inequality can also be proved by using the convexity of - log and Jensen's inequality). It is an open question to understand if such a regime, where $\kappa$ is unbounded, exists. The growth of $\int_{0}^{1} \Phi\left(f e^{(\alpha-1) C}\right) e^{-C} d v$ means that some concentration phenomenon is occuring at least in the norm measured by the entropy. Note that as far as we know, there is no Maximum Principle available for a solution of (2.1), even measured by some $L^{p}$ norm (see Section 5 below). For a better understanding of the concentration, one should further study the entropy production term

$$
\kappa^{1-\alpha} \int_{0}^{1} D(v, t) e^{(\alpha-1) C}\left|\left(f^{\alpha / 2}\right)^{\prime}+\frac{\alpha}{2} C^{\prime} f^{\alpha / 2}\right|^{2} d v
$$

It has to be noted that neither the standard logarithmic Sobolev inequality nor convex Sobolev inequalities $(\alpha \in(1,2]$, see [1]) do apply since the usual condition for the entropy - entropy production method is not satisfied.

\section{Further observations}

We conclude with some calculations which shed additional light on the properties of solutions of (2.1), (3.1), (3.2), (3.3). First consider Eqn. (2.1) without boundary conditions, i.e., $v \in \mathbb{R}$, and assume that $f$ vanishes sufficiently fast at $\pm \infty$ (this is, of course, inconsistent with traffic dynamics, where negative speeds are excluded and there are natural upper speed limits). Let $f$ be a solution of (2.1) and compute

$$
\frac{d}{d t} \int(v-u)^{2} f(t, v) d v=-2 \frac{d u}{d t} \int(v-u) f(t, v) d v+\int(v-u)^{2} \partial_{t} f(t, v) d v .
$$

The first term on the right is zero by definition of $u=u(t)$. For the second term we use the equation and emphasize the dependence of $B$ and $D$ on $v-u(t)$ as specified, for example, in (3.2), (3.3). After an integration by parts

$$
\int(v-u)^{2} \partial_{t} f(t, v) d v=2 \int B(v-u) \cdot(v-u) f d v-2 \int D(v-u) \cdot(v-u) \partial_{v} f d v .
$$


Note that for $B$ as defined by $(3.2)$ we have $B(v-u(t)) \cdot(v-u(t)) \leq 0$ with equality only if $v=u(t)$. Therefore, if $D \equiv 0$, we have $\frac{d}{d t} \int(v-u(t))^{2} f(t, v) d v \leq 0$, with equality only if $f(t, v)=\rho \delta_{u(t)}(v)$. This is consistent with the (modeling) idea that in the absence of diffusion all the drivers will brake or accelerate towards the observed average speed. In the limit $t \rightarrow \infty, u(t)$ is expected to converge towards a limit speed $u_{\infty}$ which will in general depend on $f_{0}$. The calculation leading to (6.1) remains the same if we include (zero flux) boundary conditions. Let us now consider $D \neq 0$ and $v \in \mathbb{R}$, i.e., we ignore the boundary conditions. If we formally integrate by parts in the last term in (6.1) we obtain

$$
2 \int\left[D^{\prime}(v-u(t)) \cdot(v-u(t))+D(v-u(t))\right] f(t, v) d v
$$

(plus boundary contributions if $v \in[0,1])$. Suppose for the moment that $f$ is concentrated in a small neighborhood of $u$. Then, what happens to $\int(v-u(t))^{2} f(t, v) d v$ depends on the behaviour (the sign!) of

$$
G(x):=B(x) \cdot x+D^{\prime}(x) \cdot x+D(x)
$$

with $x=v-u(t)$. As an example inspired by (3.2), (3.3), consider $D(x)=|x|^{\gamma}$ with $\gamma \in(0,+\infty)$ and $B(x)=-c x|x|$. This is actually a special case of the traffic flow model with $\delta=+\infty, c=C_{B} \rho=C_{A}(1-\rho), m_{2} \equiv 1, \sigma m_{1}(\rho)=1$. The expression (6.2) becomes

$$
-c|x|^{2}\left(|x|-\beta|x|^{\gamma-2}\right)
$$

where $\beta=(1+\gamma) / c$. Whether or not $f$ will form concentrations at $u(t)$ depends on the sign of (6.3) near $x=0$. If $G(x)>0$ for $x \approx 0$, which is the case if $\gamma<3$, then diffusion dominates and no concentrations occur. If $G(x)<0$ for $x \approx 0$ then concentrations are a possibility.

We conclude with a remark on whether or not $f$ can be expected to stay bounded (if $f$ remains bounded, so will $\kappa(t)$, and the hypotheses of Theorem 3 will apply). Assume that, at time $t, f$ assumes its maximum at $v_{0}$ (not on the boundary). By elementary calculations,

$$
\left.\partial_{t} f\right|_{v=v_{0}}+\left.B^{\prime}\left(v_{0}-u(t)\right) f\right|_{v=v_{0}}=\left.D\left(v_{0}-u(t)\right) \partial_{v}^{2} f\right|_{v=v_{0}} \leq 0 .
$$

Hence, if $v_{0} \neq u(t)$, the maximum of $f$ may grow, but its growth is controlled by $B^{\prime}\left(v_{0}-u(t)\right)$ and damped by $D\left(v_{0}-u(t)\right)$. Indefinite growth is of course consistent with the formation of concentrations. We conjecture that the solutions will actually stay bounded for all time unless concentrations form.

\section{Conclusion}

We proved the global stability of the steady solutions of a nonlinear model of traffic flow with respect to the dynamics, assuming that a quantity related to the entropy (and most probably to the absence of concentrations) stays bounded. However, in contrast to the linear case [3], this does not discard the possibility of several steady states and the asymptotic state is clearly selected by the initial conditions. Stopand-go regimes, which are observed in traffic flow experiments, are therefore not predicted by the spatially homogeneous model corresponding to (3.1), (3.2) and (3.3), at least in the asymptotic regime. However, the multivalued fundamental diagrams in combination with density fluctuations (which are, of course, excluded a fortiori in the spatially homogeneous case) should produce stop-and-go phenomena for the full Vlasov-Fokker-Planck equation. 
Acknowledgment. J.D. has been partially supported by the EU financed network no. HPRN-CT-2002-00282. This research was also supported by Grant no. 7847 of the Natural Sciences and Engineering Research Council of Canada. R.I. acknowledges the hospitality of the universities Paris 7 and 9, where most of this research was done. Both authors thank the referees for their accurate remarks which helped them to improve the presentation of their results.

\section{REFERENCES}

[1] A. Arnold, P. Markowich, G. Toscani, and A. Unterreiter, On convex Sobolev inequalities and the rate of convergence to equilibrium for Fokker-Planck type equations, Comm. P.D.E., 26:43-100, 2001.

[2] J.A. Carrillo, A. Jüngel, P.A. Markowich, G. Toscani, and A. Unterreiter, Entropy dissipation methods for degenerate parabolic problems and generalized sobolev inequalities, Monatsh. Math., 133:1-82, 2001.

[3] J. Dolbeault, R. Illner, and M. Kowalczyk, Entropy methods for explicitely time-dependent drift-diffusion equations, in preparation.

[4] R. Illner, A. Klar, and Th. Materne, Vlasov-Fokker-Planck models for multilane traffic flow, Comm. Math. Sci., 1(1):1-12, 2003.

[5] R. Illner, A. Klar, and Th. Materne, On Vlasov-Fokker-Planck type kinetic models for multilane traffic flow, to appear in Proceedings of the 23rd International Symposium on Rarefied Gas Dynamics,. Eds., Ph. Muntz and A. Ketsdever, 2003.

[6] R. Illner, C. Stoica, A. Klar, and R. Wegener, Kinetic equilibria in traffic flow models, Transport Theory Stat. Phys., 31(7):615-634, 2002.

[7] F. Otto, The geometry of dissipative evolution equations: the porous medium equation, Comm. P.D.E., 26(1-2):101-174, 2001. 